\title{
Open reduction of an old and unreduced posterior elbow dislocation
}

\author{
Tudor Mihai Gavrilă ${ }^{1,2 *}$, Emanuel Antoneac ${ }^{2}$, Cristea $\operatorname{Vlad}^{2}$, Stefan Cristea ${ }^{1,2}$ \\ ${ }^{1}$ CAROL DAVILA UNIVERSITY OF MEDICINE AND PHARMACY, BUCHAREST, ROMANIA \\ ${ }^{2}$ ST. PANTELIMON HOSPITAL, DEPARTMENT OF ORTHOPEDICS AND TRAUMATOLOGY, BUCHAREST, ROMANIA
}

\begin{abstract}
The old unreduced elbow dislocation is not very frequent, but when it is found, it is a challenge for every surgeon. We present a case of 65 years old man who came to the hospital with a dislocated elbow. After the first attempt to reduce, the elbow was mobilized in sling for 2 weeks, but during a small effort, the joint dislocated again. Another two orthopedic reduction were tried, followed by cast immobilization, but the elbow dislocated again. The patient presented in our service after two months from the injury with stiff joint in a vicious position. On imagistic examinations (Rx, CT, MRI), it was found comminuted fracture of coronoid process, posterior dislocation of olecranon and both collateral ligaments were torn. An open reduction was performed during which the joint surface was cleaned up, the anterior capsule was reattached to the coronoid process with an anchor, and then, collateral ligament was restored with the help of autograft, a gracilis muscle (bone fixed with two anchors). Postoperatively, the patient slowly began to mobilize the elbow with splint protection. After a year, the patient returned for followup; the function was completely restored and he had no pain.
\end{abstract}

\author{
Category: Case Presentation \\ Received: April 03, 2021 \\ Accepted: July 16, 2021 \\ Published: November 15, 2021 \\ Keywords: \\ old elbow dislocation, CT, MRI, open reduction, ligamentoplasty \\ Corresponding author: \\ Tudor M. Gavrilă, \\ Carol Davila University of Medicine and Pharmacy, St. \\ Pantelimon Hospital, Department of Orthopedics and \\ Traumatology, Bucharest, Romania \\ E-mail: tudor.gavrila@umfcd.ro
}

*

\section{Introduction}

Elbow dislocation is a fairly rare condition. In most cases, if the patient is young and comes to emergency room immediately, the closed reduction can be performed without further complications. If the patient neglects the dislocation and comes after a few weeks, the closed reduction can be verry difficult, even impossible, requiring open reduction and fixation of the damaged structures.

Sometimes dislocation is associated with coronoid fracture and rupture of collateral ligaments, which make the joint instable and orthopedic reduction fails. If is not operated quickly, some changes occur at the levels of cartilage, ligaments and muscle [1,2].

It is known that the metabolism of hyaline cartilage is perturbated and synovial fluid functions are altered. Because of this, the cartilage deteriorates, sometimes irreversibly. In addition, the torn collateral ligaments and muscles tendons retract, making the join impossible to reduce orthopedically. Even if the union can be reduced in a closed way, painless joint function and motion is difficult to be achieved $[3,4]$.

The are many varieties of elbow dislocations, the most common being the posterior ones. In this case, the arm is fixed in extension and the range of motion is very limited. Pronation and supination are also reduced in amplitude. Because the biceps tendon is under tension over the humeral condyles, the forearm is fixed in supination, making pronation almost impossible.

The old dislocation is characterized by many other changes, such as shortening of the triceps muscle and collateral ligaments, tightening of ulnar nerve, fibrous tissue formation in olecranon, coronoid fossa and in space between radial head and distal end of humerus, thickening of joint capsule and ossification of muscles (especially brachioradialis, triceps and biceps brachii) $[5,6]$.

Ossification is practically the result of the biological transformation of the local hematoma. Some authors 
describe a" radial humeral horn", an ossification developed near the radial head under the periosteum and capsule [7].

\section{Case Presentation}

We present a 65-year-old man with an unreduced and unstable old dislocation of his right elbow. He fell on this forearm and dislocated the join. In the emergency-room the dislocation was reduced orthopedically and immobilized in a sling for two weeks. After this period, he started the motion, but the joint dislocated again. Any attempt to reduce the dislocation failed, so he came in our hospital after 2 months after the injury.

On physically examination, the elbow was stiff, with the forearm in supination and very slight flexion. All the muscles were in tension, the pronation was impossible and the anatomy of the region was modified, the olecranon being preeminent posterior under the skin. Fortunately, he had no neurological changes, and the pulse at the radial artery was present.

Plain radiographs showed posterior dislocation of the elbow (Figure 1, a-b). Preoperatively, a CT scan with reconstruction was performed to better visualize the joint. The coronoid process was fractured and the distal end of the humerus was displaced anteriorly in its place (Figure 2, a-b). The patient did not complain of too much pain, but the function was severely impaired.
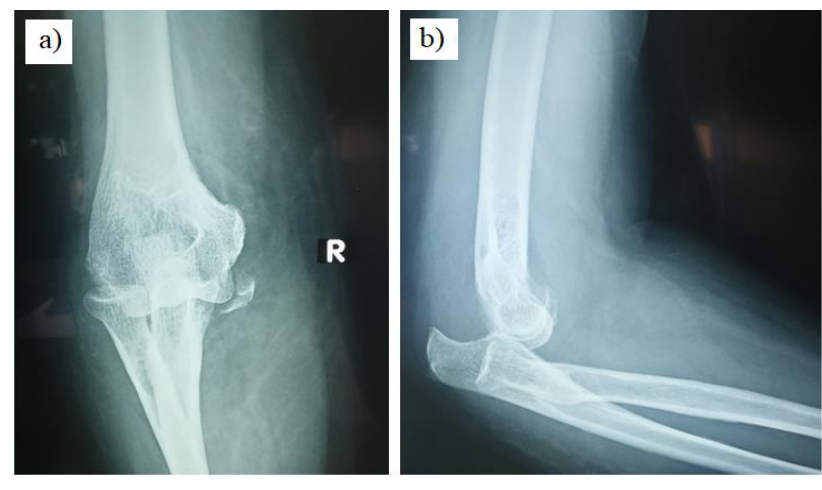

Figure 1. a) The anteroposterior radiography; b) The lateral radiography shows posterior dislocation of elbow

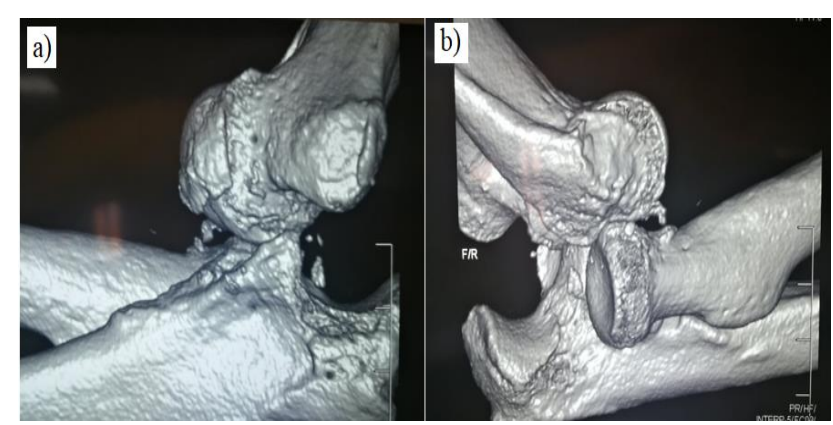

Figure 2. a) The fracture of coronoid process and anterior displacement of distal humerus can be seen on CT reconstruction image; b) Posterior displacement of olecranon
After a thorough analyze of the case, we made the decision to open the joint to reduce and stabilize the elbow. With the patient in supine position, we harvested the gracilis tendon from the same side of the knee. Then, we made an incision over posterior aspect of the radial head, prolonged over the lateral side of lateral epicondyle (Kocher approach) [8,9].

The lateral extensors were detached and then the joint was carefully inspected. All fibrous tissues were removed from the coronoid and olecranon fossa. During the process, the torn collateral ligament insertion was detached from the bone to allow reduction of the elbow and complete movement.

After debridement, the distal humerus was put in his place, but the joint still remained instable in the anteroposterior plane. The anterior capsule was attached to the coronoid process with an anchor (Figure 3). After this, the flexion-extension movement was checked. With the elbow antero-posterior stabilized, we focused on lateral instability. A drill hole was made near the isometric center of the epicondyle. The tendon was bent in two with the help of PushLock anchor, one end of the tendon being attached to the epicondyle.

A new hole was made in the ulna and the other end of bent tendon was inserted and attached to the bone with another PushLock. Stability in the lateral plan and elbow motion was verified. After that, the extensor muscles were sutured to the lateral edge of the humerus. The soft tissues were closed with a 3-0 Vicryl and the skin with 3-0 Prolene.

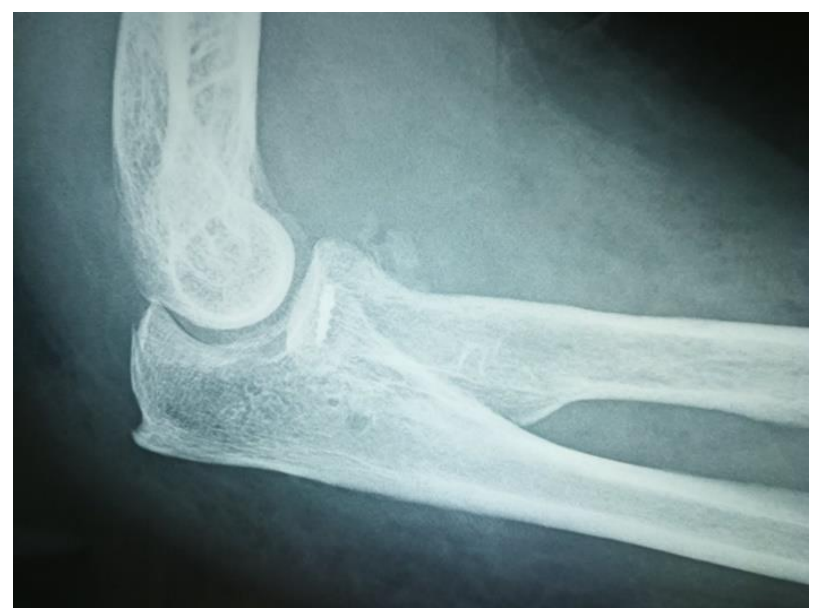

Figure 3. Metallic anchor that attaches the anterior capsule to the coronoid process

The arm was immobilized in a posterior splint in the elevated position. Splint was removed several times a day to allow gently flexion-extension and pronation-supination movements. With the rage of the movement and the regained strength, the splint was discarded during the day, but was worn during the night for 2-3 months. After a year, the patient returned for follow-up (Figure 4, a-d). 


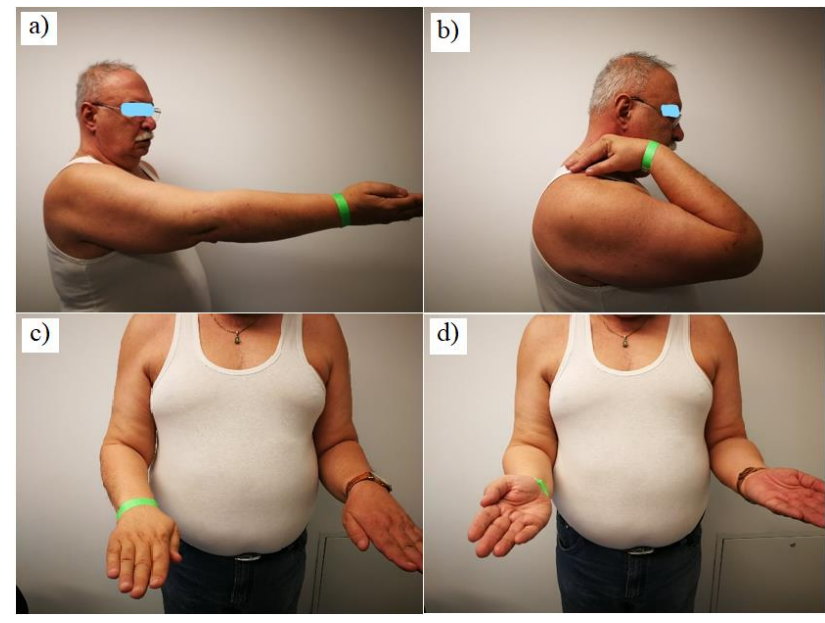

Figure 4 (a-d). Patient range of motion after one year from surgery.

\section{Discussion}

Elbow is a joint who consists of three bones: humerus, ulna and radius. Two strong ligaments (ulnar collateral ligament and lateral collateral ligament) ensure the stability of the joint. In severe trauma, these ligaments are torn and the distal end of the humerus can lose its place, moving in most cases anteriorly.

Usually, elbow dislocation is treated orthopedically, with a close reduction followed by splint immobilization for two weeks, time required for healing of injured ligaments. After that period, the splint is removed and the patient starts the progressive movement of the elbow.

If the dislocation is associated with the fracture of the coronoid process, the joint remains instable. A joint who stays unreduced for more than 3 weeks becomes stiff, being almost impossible to be reduced by orthopedic maneuvers $[10,11]$. In our case we have an old man with unreduced right elbow dislocation. In addition to dislocation, the fracture of the coronoid process makes the joint unstable. First attempts of reduction failed and he remained for two months in a splint. Many changings occurred during this period. The cavity of joint filled with fibrous tissue, the capsule became thick, the tendons around the elbow, as triceps, biceps, brachialis were tensioned and shortened, making pronation very limited with stiffness in supination.

In this case, the only therapeutic option was open reduction of dislocation associated with reconstruction of the lateral collateral ligament. Imagistic examinations (radiographs, CT and MRI) are mandatory and showed us in detail bone deficiency and pattern of dislocation, thus helping to plan the operation. In particular, CT reconstruction was absolutely necessary to detect the bone lesions and displacements.

With the patient in supine position, the gracilis tendon was first harvested from the knee, this autograph being necessary for the reconstruction of lateral collateral ligament; then through a lateral approach, we opened the joint. After a thorough examination and debridement, the bones were relocated and the anterior capsule was attached to the coronoid process with a metallic anchor. With elbow stabilized in the antero-posterior plane, the lateral instability was treated. The Gracilis tendon was used to reconstruct the torn lateral collateral ligament.

Postoperatively, the surgery was protected with a splint. Immediately after a few hours, the splint was removed and the patient started gently active of motion. Rehab program helped the patient to regain strength and complete range of motion. Thus, a year after the operation, he was able to have full extension and flexion, full pronation and a very slow deficit of supination. It is known that for daily activity, a range of 100-degree motion is enough, but our patient had restored almost full motion.

\section{Highlights}

$\checkmark$ Old unreduced elbow dislocation is a rare condition, but the pathology is very difficult to treat.

$\checkmark$ Open reduction, debridement of the joint surfaces, fixation of torn capsule and reconstruction of collateral ligament with gracilis (or allograph) is the most common procedure.

\section{Conclusions}

Despite its rarity, elbow dislocation must be recognized and treated quickly, the most common method being close reduction. An acute dislocation can be reduced quite easily, with good long-term results.

Imagistic investigations are mandatory to discover associated bone lesions that may influence the prognostic. A fracture of coronoid process can lead to instability, making the close reduction to fail.

The old unreduced dislocation is followed by multiple changes inside the joint, such as fibrosis, shortening of muscles, tension of ulnar nerve, stiffness in vicious positions. All these pathological modifications make the close reduction impossible $[12,13]$.

In the case of an old unreduced dislocation, the only option is to open the joint and to fix all damaged structures inside $[14,15]$.

A thorough understanding of the anatomy and function of the elbow is necessary to perform such an operation. Reconstruction of collateral ligament is a very important step for a successful result. For this reason, such an operation performed in special units with personnel trained to address this pathology.

Surgery is not everything in such cases. Postoperatively, a careful rehabilitation program helps the patient to restore his elbow function. To obtain a good result, it is necessary to alternate between the splint protection and the active/ passive range of motion. 
The patient compliance is important, so the doctor must explain in detail the main steps of the surgery and what he expects to do after the operation. Close collaboration between the patient, his doctor and the rehabilitation team is the key to regaining his elbow function.

\section{Conflict of interest disclosure}

There are no known conflicts of interest in the publication of this article. The manuscript was read and approved by all authors.

\section{Compliance with ethical standards}

Any aspect of the work covered in this manuscript has been conducted with the ethical approval of all relevant bodies and that such approvals are acknowledged within the manuscript.

\section{References}

1. Prasetia R, Mahyudin, Rasyid HN. Open Reduction on very late-presenting unreduced posterior elbow dislocation: Still promising treatment option. Int J Surg Case Rep. 2021 Jul;84:106056. doi: 10.1016/j.ijscr.2021.106056

2. O'Brien AC, Teh Z, Rinaldi M, Lee E, Hughes R, Aktselis I, McKean D. Intraosseous Type 2 Median Nerve Entrapment After Posterior Elbow Dislocation Diagnosed on Ultrasound With MRI and Surgical Correlation. Cureus. 2021 Oct 8;13(10):e18606. doi: 10.7759/cureus. 18606

3. Reichert IL, Ganeshamoorthy S, Aggarwal S, Arya A, Sinha J. Dislocations of the elbow - An instructional review. J Clin Orthop Trauma. 2021 Jul 13;21:101484. doi: 10.1016/j.jcot.2021.101484.

4. van der Horst AS, Stephens AR, Wei G, Presson AP, Tashjian RZ, Kazmers NH. Prognostic Factors Affecting Long-Term Outcomes After Elbow Dislocation: A Longitudinal Cohort Study. J Hand Surg Glob Online. 2021 Sep;3(5):260-265. doi: 10.1016/j.jhsg.2021.05.011

5. Tudorachi NB, Eva I, Dascalu CG, AL-Hiary R, Barbieru B, Paunica M, Motofei C, Moraru AC. The influence of serum calcium and magnesium levels in the radiological evolution of knee osteoarthritis. J Mind Med Sci. 2020;7(2):217-226. doi: 10.22543/7674.72.P217226
6. Gavrilă MT, Cristea S, Antoneac E. Study on the arthroscopic repair of the rotator cuff tear. J Clin Invest Surg. 2019;4(1):38-41. doi: 10.25083/2559.5555/4.1/38.41

7. Islam S, Jahangir J, Manzur RM, Chowdury AA, Tripura N, das A. Management of neglected elbow dislocations in a setting with low clinical resources. Orthop Surg. 2012 Aug;4(3):177-81. doi: 10.1111/j.1757-7861.2012.00190.x

8. Pundkar GN, Sonar SB, Kulkarni OP. A Case of 3month-old Neglected Elbow Dislocation Managed by Open Reduction and Cruciate Ligament Reconstruction Using Tendon Graft. J Orthop Case Rep. 2017 JulAug;7(4):58-60. doi: 10.13107/jocr.2250-0685.852

9. Coskun HS, Usta MB, Say F, Yildirim AM. Factors affecting post-traumatic stress disorder in children with orthopedic injury. J Clin Invest Surg. 2020;5(1):24-28. doi: 10.25083/2559.5555/5.1/24.28

10. Arafiles RP. Neglected posterior dislocation of the elbow. A reconstruction operation. J Bone Joint Surg Br. 1987 Mar;69(2):199-202. doi: 10.1302/0301620X.69B2.3546327

11. Vreju FA, Ciurea PL, Chisalau BA, Parvanescu CD, Firulescu SC, Turcu-Stiolica A, Barbulescu AL, Dinescu SC, Dumitrescu CI, Cristina C, Radu L, Dumitrescu D. The effect of glucosamine, chondroitin and harpagophytum procumbens on femoral hyaline cartilage thickness in patients with knee osteoarthritisAn MRI versus ultrasonography study. J Mind Med Sci. 2019;6(1):162-168. doi: 10.22543/7674.61.P162168

12. Naidoo KS. Unreduced posterior dislocations of the elbow. J Bone Joint Surg Br. 1982;64(5):603-6. doi: 10.1302/0301-620X.64B5.7142268

13. Kanakaraddi S. Primary total elbow replacement in a patient with old unreduced complex posterior elbow dislocation. Bull Hosp Jt Dis. 2013;71(4):294-6.

14. Concina C, Crucil M, Theodorakis E, Saggin G, Perin S, Gherlinzoni F. Complex open elbow fracturedislocation with severe proximal ulna bone loss: a case report of massive osteochondral allograft surgical treatment. Clin Shoulder Elb. 2021 Sep;24(3):183-188. doi: 10.5397/cise.2021.00220

15. Al-Ani Z, Wright A, Ricks M, Watts AC. The threecolumn concept of elbow joint stability and the Wrightington elbow fracture-dislocation classification, emphasizing the role of cross-sectional imaging. Emerg Radiol. 2021 Aug 17. doi: 10.1007/s10140-021-01978-3 